\title{
A longitudinal study of in vitro tests for lymphocyte function in rheumatoid arthritis
}

\author{
JOHN S. PERCY, PAUL DAVIS*, ANTHONYS. RUSSELL*, AND \\ ESTELLE BRISSON
}

From the Rheumatic Disease Unit, Department of Medicine, University of Alberta, Edmonton, Alberta, Canada

SUMMARY In vitro tests of lymphocyte function have been performed in 61 patients with 'classical' $\omega$ or 'definite' rheumatoid arthritis. In vitro lymphocyte function was assessed by lymphocyte trans-ir formation responses to phytohaemagglutinin (PHA), Pokeweed mitogen (PWM), Candida antigen, $\frac{\oplus}{\oplus}$ and herpes simplex type I (HSV1). Follow up data were available after 6 months of treatment in $32^{\circ}$ of these patients. Spontaneous lymphocyte transformation was assessed in all patients. Results obtained in patients with rheumatoid arthiritis were compared to those seen in a normal control population. Disease activity of patients with rheumatoid arthritis was assessed using standard clinical methods.

Lymphocytes from patients with rheumatoid arthritis showed a similar degree of spontaneous transformation to that seen in normal subjects. In contrast, lymphocytes from patients with rheumatoid arthritis responded less well to PHA and Candida and HSV1 antigens when compared to normal patients. In patients with rheumatoid arthritis the response to PWM was markedly enhanced compared to normals. Clinical improvement was noted in 19 of the 32 patients seen at follow up, all of whom had received gold or penicillamine therapy. The abnormal responses of PHA and PWM seen before treatment became normal in those patients who improved clinically. The responses to Candida and HSV1 antigens not only returned to normal following treatment but were increased above those seen in normal controls. A statistically significant association was seen between clinical improvement and improvement of in vitro tests of lymphocyte function.

Classical rheumatoid arthritis is characterised histologically by the presence of rheumatoid granulomata which can be regarded as a manifestation of a cellmediated immune response directed against an, as yet unknown, antigen. Previously published observations on cell-mediated immunity in rheumatoid arthritis are conflicting (Menard et al., 1973; Waxman et al., 1973; Lance and Knight, 1974; Messner, 1974; Sheldon et al., 1974; Hepburn et al., 1976). Some studies show a normal response to PHA while others show that the response to this and other mitogens and antigens is impaired. One report demonstrated an increased rate of spontaneous transformation in lymphocytes from patients with rheumatoid arthritis (Menard et al., 1973). One possible explanation for the discrepant results

\section{Accepted for publication January 3, 1978}

Correspondence to $n_{r}$. J. S. Percy, 9-112 Clinical Sciences Building, Universit, of Alberta, Edmonton, Alberta, Canada. * Paul Davis and Anthony S. Russell are Associates of the Canadian Arthritis and Rheumatism Society. previously reported is that the majority of studies have been made without sequential observations and in addition little attempt has been made to correlate abnormal responses to the patient's clinical state. We have carried out a longitudinal study on patients with rheumatoid arthritis to determine whether or 3 not a correlation exists between some in vitro tests $\frac{D}{O}$ of lymphocyte function and clinical disease activity.

\section{Patients and methods}

Sixty-one patients with ARA 'classical' or 'definite' $\omega$ rheumatoid arthritis were studied. In 32 of these patients observations were made before the institu- $\stackrel{0}{\simeq}$ tion of either gold or penicillamine therapy and again after 6 months of treatment. Rheumatoid disease activity was assessed by measurement of $\frac{T}{0}$ handgrip strength, aggregate proximal interphalan- $\underset{\mathbb{D}}{\mathbb{D}}$ geal joint circumference, duration of morning $\frac{\mathrm{P}}{\mathrm{D}}$ stiffness, haemoglobin level, and eryrthrocyte sedi- $\stackrel{\mathbb{D}}{2}$ mentation rate. Gold was administered in the form 
of $50 \mathrm{mg}$ Myochrysine intramuscularly at weekly intervals for the first 20 weeks and at monthly intervals thereafter. D-penicillamine was given in a dosage of $250 \mathrm{mg}$ daily with monthly increments of $250 \mathrm{mg}$ to a maintenance dosage of $250 \mathrm{mg}$ tid. At the time when clinical observations were made blood samples were drawn for in vitro testing of lymphocyte function. Control studies were performed on blood taken from 30 apparently healthy volunteers.

Lymphocytes were separated from whole blood by a standard Ficoll Hypaque method. Lymphocyte concentrations were adjusted to $1 \times 10^{6}$ cells $/ \mathrm{ml}$. for PHA and PWM responses and to $2 \times 10^{6}$ cells $/ \mathrm{ml}$ for HSV1 and Candida. Cells were suspended in RPMI 1640 (Grand Island Biological Co., Burlington, Ontario, Canada). Cell suspensions were distributed into flat bottomed microtitre plates. Mitogens or antigens were added to cell suspensions in triplicate. The cells were cultured for 144 hours for both mitogens and antigens. Six hours before harvesting $10 \mu l$ of ${ }^{3} \mathrm{H}$ thymidine was added to the cultures (concentration $20 \mu \mathrm{Ci} / \mathrm{ml}$, specific activity $40 \mathrm{mCi} / \mathrm{mmol}$ ). Viability of cultures was routinely determined before harvesting which was performed using a multisample semi-automated harvester (Skatron, Lierbyen, Norway).

Cultures of unstimulated cells from the same patient were made at all times in order to determine the rate of spontaneous transformation. Maximal stimulation responses were obtained by selecting optimum doses of both mitogens and antigens by preparing dose response curves. Doses of antigens and mitogens were tested in the following ranges:PHA (Difco Laboratories, Detroit, Michigan, USA) $0-100 \mu \mathrm{g} / \mathrm{ml}$-mean optimum dose $14 \mu \mathrm{g} / \mathrm{ml}$; PWM 0-50 $\mu \mathrm{g} / \mathrm{ml}$-mean optimum dose $25 \mu \mathrm{g} / \mathrm{ml}$; Candida antigen (Hollister Stier Laboratories, Missisauga, Ontario, Canada) 0-1:5000 dilution stock solution-mean optimum dosage 1:2000, The HSV1 antigen was prepared by treatment of an infected monkey cell culture (Vero) with $0.3 \%$ propiolactone (Russell, 1974).

After harvesting, the fibre discs were removed, placed in $10 \mathrm{ml}$ of scintillation fluid and the emissions counted in a Beckman L.S. 230 scintillation counter. Results were expressed as the long of the ratio be:tween stimulated and unstimulated cells from the same subject.

\section{Statistics}

The significance of lymphocyte responsiveness to mitogens and antigens comparing normals against pretreatment rheumatoid patients, normals versus post-treatment rheumatoid patients, and rheumatoid patients pretreatment versus post-treatment was calculated using sample tests of proportions. The relationship between laboratory variables to clinical status was tested using binomial distribution tables with a probability of success of $\mathbf{0 . 5}$.

\section{Results}

\section{SPONTANEOUS TRANSFORMATION}

A wide range of spontaneous transformations was seen in patients with rheumatoid arthritis and in normal controls. No statistically significant difference was detected between the results obtained in both groups.

\section{LYMPHOCYTE RESPONSIVENESS TO MITOGENS}

The PHA responses of lymphocytes in patients with rheumatoid arthritis was significantly less than the PHA response in the 30 normal control subjects (Fig. 1). Only 2 normal subjects had a $\log _{10}$ transformation index of less than 1 . Seventeen patients with rheumatoid arthritis had a depressed response

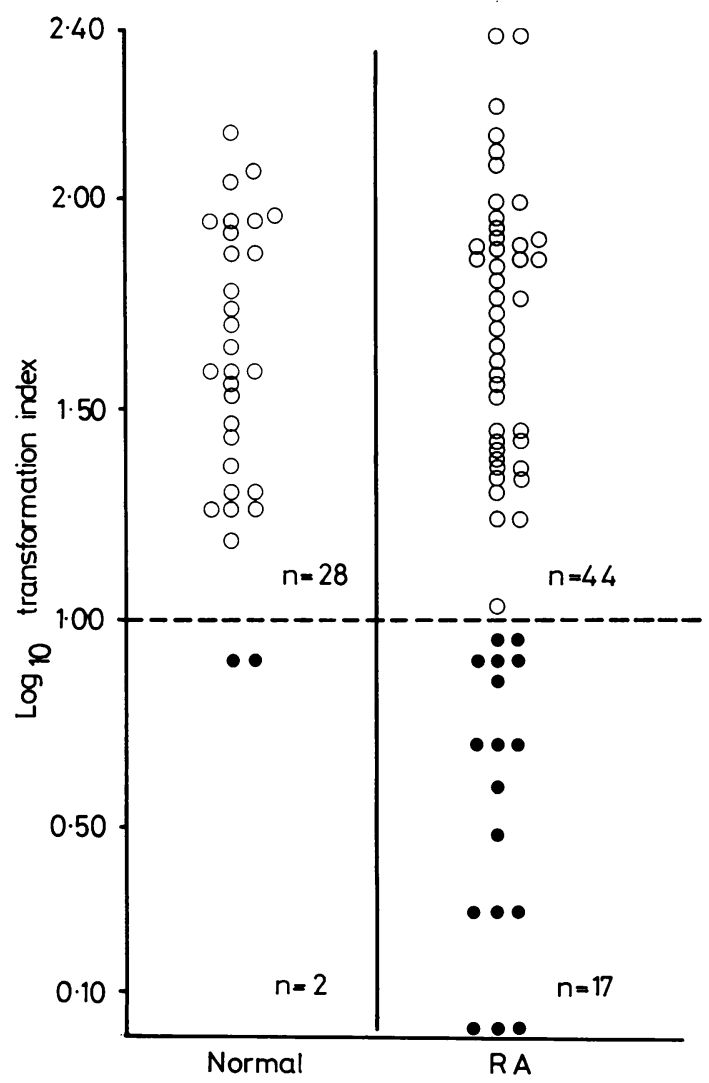

Fig. 1 Log 10 transformation indices to $P H A$ in 61 randomly selected patients with $R A$ compared to 30 normal controls 
$(P<0 \cdot 01)$. Follow up data were available after 6 months' treatment with either gold or penicillamine in 33 patients and there was a trend towards normalisation of the responses in patients with rheumatoid arthritis. At this time only 5 of 33 patients had a depressed PHA response. There was a statistically significant difference $(P<0.05)$ between PHA responses seen in patients before treatment and after treatment (Fig. 2). There was no significant difference between PHA responses in the normal group and in the 33 patients seen with rheumatoid arthritis at follow up. Patients with rheumatoid arthritis had lymphocyte transformation responses to PWM which were significantly elevated $(P<0.001)$ over those levels seen in normal control subjects (Fig. 3). At follow up there had been a normalisation of the PWM response in the 32 patients studied which was significantly different $(P<0.001)$ from pretreatment results (Fig. 4). There was no statistically significant difference between PWM response of the normal control population and the 32 patients seen

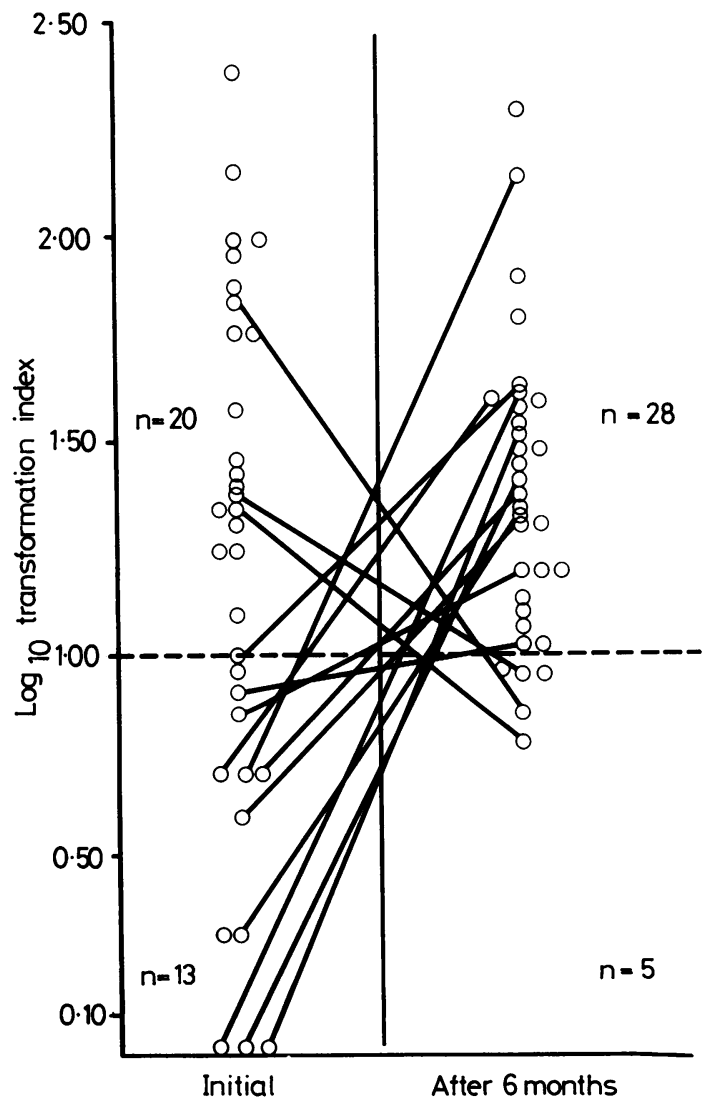

Fig. 2 Changes in $P H A$ responses seen in 33 patients with $R A$ initially and at 6 month follow up with rheumatoid arthritis at follow up. Successful?

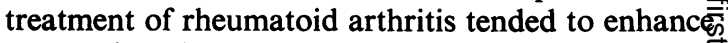
in vitro lymphocyte responsiveness to PHA whereas the response to PWM was reduced.

LYMPHOCYTE RESPONSES TO ANTIGENS Lymphocyte responses to HSV1 and Candida antigens in patients with rheumatoid arthritis were reduced when compared with normal controls $(P<0.05)$. At follow up the 31 patients seen after 6 months' treatment with gold or penicillamine demonstrated an increase in the lymphocyte re욜 sponsiveness to these 2 antigens and a significan difference $(\mathrm{P}<0 \cdot 01)$ existed between responses before and after treatment. A striking feature was that the post treatment response to Candida and HSV1 was enhanced when compared to the normal controb group.

RELATIONSHIP BETWEEN CLINICAL IMPROVEMENT AND IMPROVEMENT IN LABORATORY VARIABLES

Of the 61 patients with rheumatoid arthritis seeng initially, 32 patients were available for follow upo after 6 months' treatment with either gold or penie cillamine therapy. At this time 19 patients had show clinical improvement and 13 remained unchanged Of the 12 clinically improved patients whose initiad response to PHA was depressed 11 showed improve ment to normal levels at follow up (Fig. 5). This

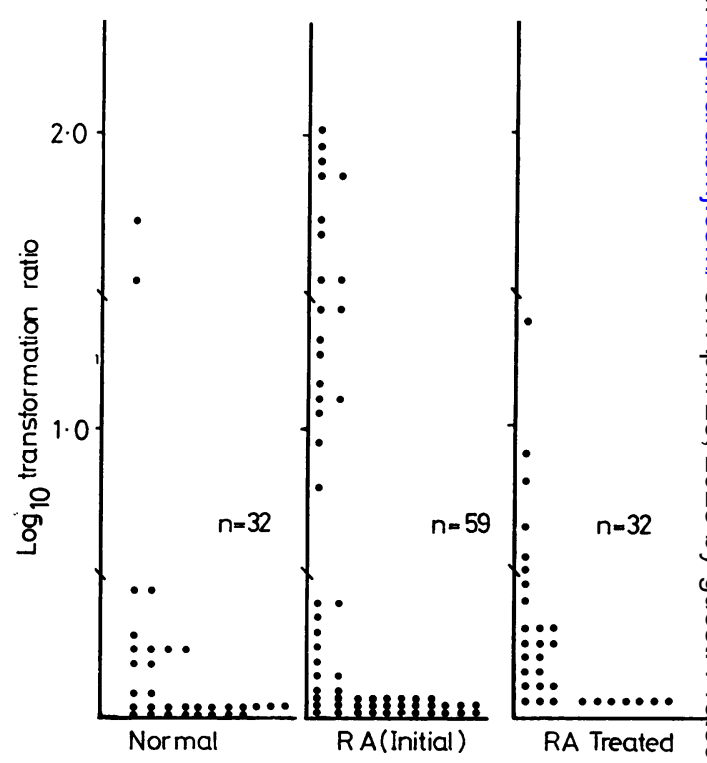

Fig. 3 Comparison of $P W M$ transformation indices in 32 normal controls, 59 randomly selected patients with $R A$, and 32 of these patients seen at follow up 


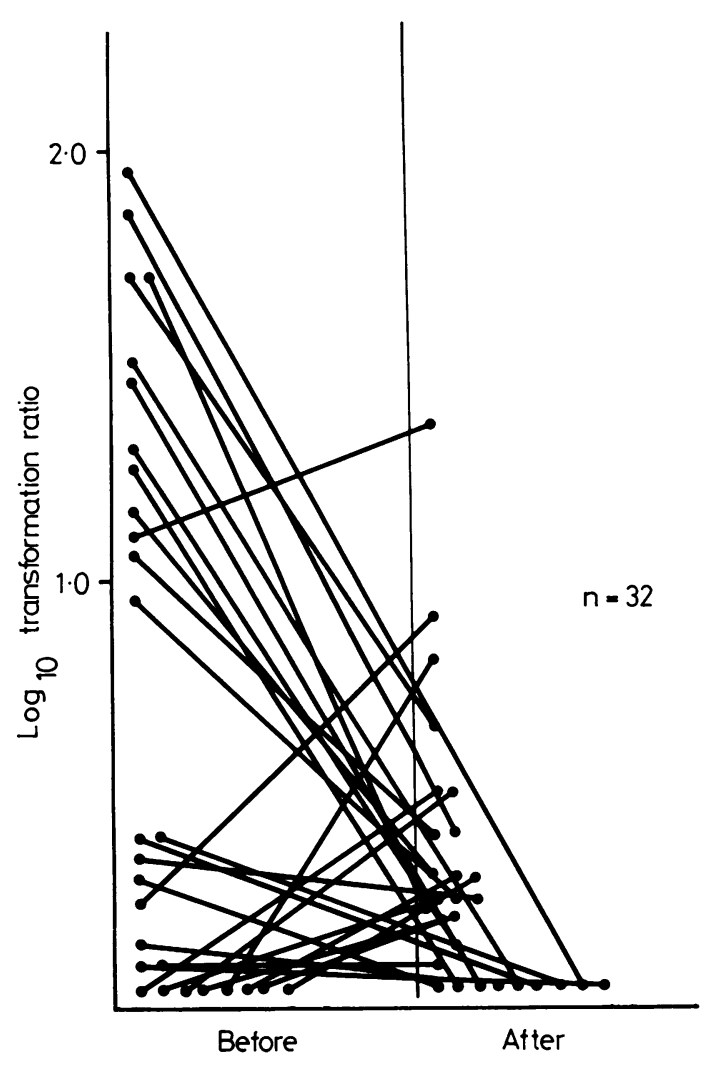

Fig. 4 Changes in $P W M$ responses in 32 patients with $R A$, initially and at 6 month follow up

correlation is significant $(\mathrm{P}<0.05)$. In contrast, in the clinically unchanged group improvement in PHA response occurred in 2 out of the 3 patients in whom it had been suppressed (Fig. 6). Similar changes were seen in response to Candida and HSV1 antigens $(\mathrm{P}<0.05)$. The PWM response tended to decline to normal levels with clinical improvement $(\mathrm{P}<0 \cdot 05)$.

\section{Discussion}

The results of our study support those previously published which suggest that in vitro lymphocyte responsiveness to both mitogens and antigens is abnormal in patients with rheumatoid arthritis (Menard et al., 1973; Waxman et al., 1973; Lane and Knight, 1974; Messner, 1974; Sheldon et al., 1974; Hepburn et al., 1976). In addition, by means of a longitudinal study in 32 of the 61 patients originally studied, we have been able to demonstrate that patients receiving treatment undergo normalisation of their lymphocyte responsiveness to both mitogens nd antigens and that this shows good correlation

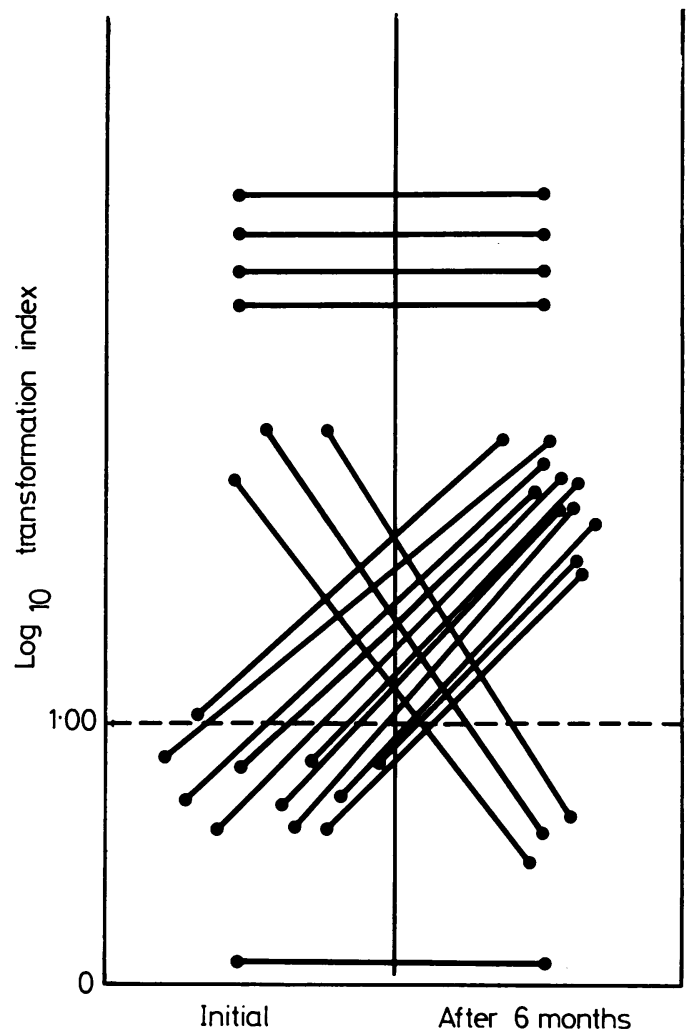

Fig. 5 Changes in PHA responses, initially and at 6 month follow up, in the 19 patients with $R A$ who showed clinical evidence of improvement

with improvement in the patients' clinical state. We have been unable to confirm the previous report that spontaneous lymphocyte transformation is enhanced in patients with rheumatoid arthritis (Menard et al., 1973). Any group of patients suffering from rheumatoid arthritis will show a great range of disease activity and this may indeed account for some of the apparent discrepancies between previous reports of studies of lymphocyte function in rheumatoid arthritis. Our initial group of patients consisted of 61 patients taken at random from our clinics. Although this group contained patients who had been on long-term management for their disease and were in clinical remission, an attempt was made to include in this group all patients with newly diagnosed rheumatoid arthritis before institution of therapy other than routine anti-inflammatory drugs. Thus a considerable number of patients in our study had active disease at the time of their initial evaluation. At follow up every attempt was made to include patients who had previously been seen with active disease and in the interim period had received treatment with either gold or penicillamine. Included 


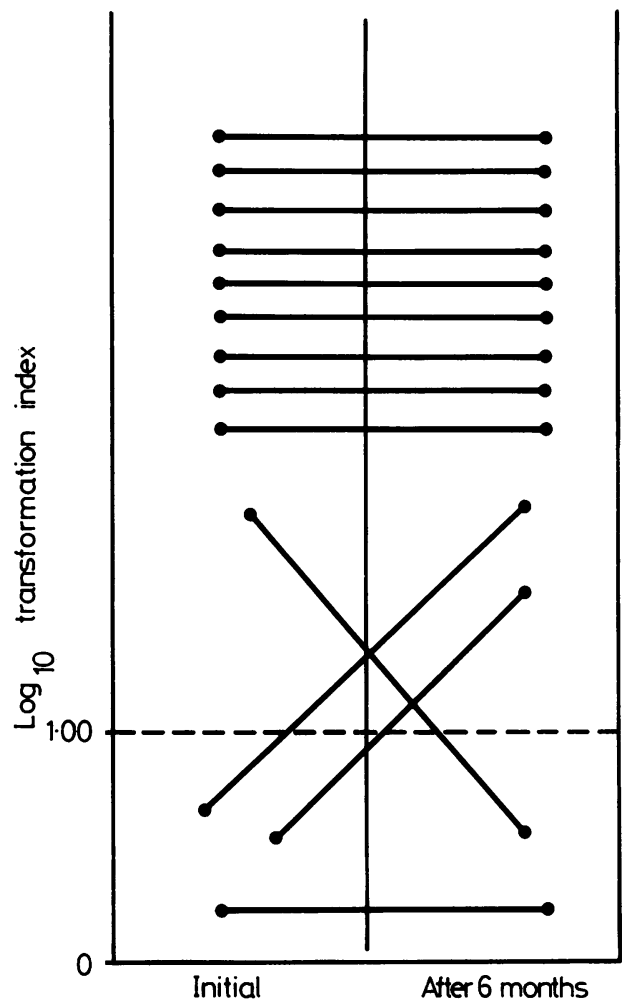

Fig. 6 Changes in PHA responses, initially and at 6 month follow up, in the 13 patients with $R A$ who were clinically unchanged throughout the study

in this group, however, were also some patients who had been in clinical remission throughout the 6 months' period. It is therefore important to note that in our study, improvement in laboratory variables of lymphocyte function in rheumatoid arthritis appeared to relate to the institution of active therapy for rheumatoid arthritis. In particular, those patients who were studied sequentially tended to be suffering from severe progressive disease which required either the institution of either gold or penicillamine therapy. Thus the abnormal lymphocyte response to PHA, Candida, and HSV1 antigens in patients in this group may reflect the severity of their disease, as may indeed be the enhanced response seen to PWM. Clinical improvement was associated with normalisation of all these responses and in the case of both Candida and HSV1 a marked enhanced response was seen following treatment. It is impossible to determine that this improvement in in vitro tests of lymphocyte function is the direct effect of the institution of gold or penicillamine therapy or a direct consequence of the patient's overall clinical improvement. The possibility that gold and penicillamine may have an effect on lymphocyte function which is independent of their effects on rheumatoid disease activity exists. We have $\stackrel{\vec{S}}{\vec{S}}$ been unable to produce any in vitro evidence of this 0 although Harth et al. (1976) has been able to dem-흠 onstrate an apparent suppression of lymphocyte re- $\overline{\overline{\bar{n}}}$ sponse to PHA after incubation with gold salts in vitro.

The interpretation of the in vitro responsiveness of lymphocytes to PWM and other antigens in $\operatorname{man}_{-}^{\infty}$ is the subject of some debate. The PHA response? appears to be largely a reflection of T-cell function. $\overrightarrow{\vec{\omega}}$ PWM may also be an indicator of B-cell activity. T $\stackrel{\sim}{\omega}$ cells are probably primarily responsible for the reaction to Candida and HSV1 antigens. If this interpretation is applied to our data, the results are ${ }_{i r}$ fully congruous in that restoration of T-cell function as reflected by improvement in PHA response is $\vec{\sigma}$ associated with a return towards normal of the PWM 은 response. Improvement in T-cell function could also be held to account for the increase in magnitude of $O$ the response to HSV1 and Candida antigens.

Our study would suggest that patients with active $\frac{O}{8}$ rheumatoid arthritis do have significantly impaired lymphocyte responses as tested in in vitro methods $\overrightarrow{0}$ and that this abnormality is directly related to 0 clinical disease state. These abnormal responses appear to be reversible and after 6 months' follow up in a longitudinal study we have been able to demonstrate an improvement in T-cell function which is associated with clinical improvement to the extent $\frac{}{\circ}$ that patients with inactive rheumatoid arthritis had $\overrightarrow{\vec{B}}$ responses indistinguishable from those seen in $\frac{3}{3}$ normal controls.

\section{References}

Harth, M., Stiller, C. R., Evans, J., Zuberi, R., McGirr, D., 으 and Sinclair, N. (1976). Effects of sodium aurothiomalate $\frac{O}{3}$ on human lymphocyte functions in normal controls and patients with rheumatoid arthritis. (Abstract.) Arthritis and Rheumatism, 19, 802.

Hepburn, B., McDuffie, F. C., and Ritts, R. E. (1976). Impaired blastogenic response of lymphocytes from synovial fluid and peripheral blood of patients with rheumatoid $D$ arthritis. Journal of Rheumatology, 3, 118-122.

Lance, E. M., and Knight, S. C. (1974). Immunologic reactivity in rheumatoid arthritis. Arthritis and Rheumatism, 17, $\mathrm{N}$ 513-520.

Menard, H. A., Haim, T., and Peltier, A. P. (1973). In vitro studies on peripheral blood leucocytes in rheumatoid arthritis. Annals of the Rheumatic Diseases, 33, 361-365.

Messner, R. P. (1974). Clinical aspects of T- and B-lymphocytes in rheumatic diseases. Arthritis and Rheumatism, 17, 339-346.

Russell, A. S. (1974). Cell-mediated immunity to herpes simplex virus in man. Journal of Infectious Diseases, 129, 142-146.

Sheldon, P. J., Papamichail, M., and Holborow, E. J. (1974). Studies on synovial fluid lymphocytes in rheumatoid arthritis. Annals of the Rheumatic Diseases, 33, 509-513.

Waxman, J., Lockshin, M. D., Schnapp, J. J., and Doneson, I. N. (1973). Cellular immunity in rheumatic diseases Arthritis and Rheumatism, 16, 499-506. 